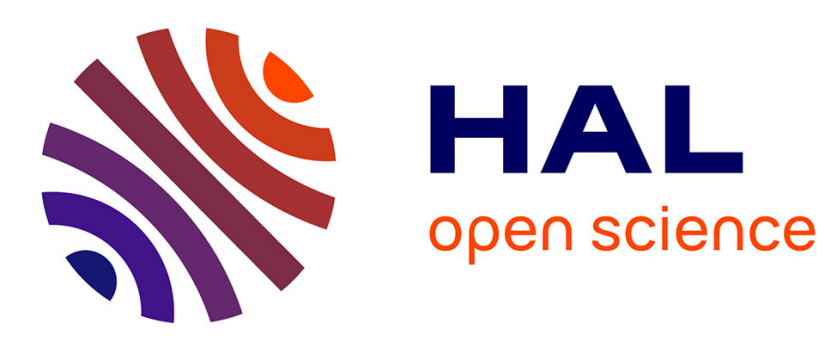

\title{
Supermolecular switches based on multiwalled carbon nanotubes
}

Arunkumar Subramanian, Lixin Dong, Bradley J.Nelson, Antoine Ferreira

\section{To cite this version:}

Arunkumar Subramanian, Lixin Dong, Bradley J.Nelson, Antoine Ferreira. Supermolecular switches based on multiwalled carbon nanotubes. Applied Physics Letters, 2010, 96 (073116), pp.1-3. 10.1063/1.3327514 . hal-00648516

\section{HAL Id: hal-00648516 https://hal.science/hal-00648516}

Submitted on 5 Dec 2011

HAL is a multi-disciplinary open access archive for the deposit and dissemination of scientific research documents, whether they are published or not. The documents may come from teaching and research institutions in France or abroad, or from public or private research centers.
L'archive ouverte pluridisciplinaire HAL, est destinée au dépôt et à la diffusion de documents scientifiques de niveau recherche, publiés ou non, émanant des établissements d'enseignement et de recherche français ou étrangers, des laboratoires publics ou privés. 


\title{
Supermolecular switches based on multiwalled carbon nanotubes
}

\author{
A. Subramanian ${ }^{\text {a) }}$, L. X. Dong ${ }^{\text {b) }}$, B. J. Nelson ${ }^{\text {c) }}$
}

Institute of Robotics and Intelligent Systems, ETH Zurich, 8092 Zurich, Switzerland

A. Ferreira

Instutit PRISME, ENSI Bourges, 88 Boulevard Lahitolle, 18000 Bourges, France

${ }^{\text {a) }}$ Presently at Center for Integrated Nanotechnology, Sandia National Laboratories, Albuquerque, NM 87185

${ }^{b}$ Presently at Department of Electrical and Computer Engineering, Michigan State University, East Lansing, MI 48824

${ }^{c)}$ Electronic mail: bnelson@ethz.ch

\begin{abstract}
Electrostatically actuated nanoelectromechanical switches based on inter-shell displacement mechanisms within batch fabricated, bi-directional multiwalled carbon nanotube (MWNT) bearings are reported. Multiple devices with a 220nm pitch are constructed within individual MWNT supermolecules. Experimental results on performance metrics including low switching voltages $(0.8$ to $6 \mathrm{~V})$, repeatability, hysteresis and failure modes are presented.
\end{abstract}


Arc-grown multiwalled carbon nanotubes (MWNTs) represent a physical realization of ideal building blocks for nanoelectromechanical systems (NEMS). Suitable modification of the asgrown, nested-shell MWNT architecture makes it possible to slide ${ }^{1,2}$ and rotate ${ }^{3}$ individual nanotube shells with respect to each other. The high-speed, low friction, low fatigue and low wear characteristics of these linear / rotary nanobearings have been exploited to create nanomotors $^{3-5}$ and nanoresonators. ${ }^{6-7}$ Electromechanical switches, relays, memories and logic elements represent another important family of devices that can be realized using telescoping nanobearings. ${ }^{8-13}$ These devices compare favorably with other technologies in terms of size, power consumption and speed (estimated to be in the $\mathrm{GHz}$ regime). However, investigations of these electrostatically actuated telescoping devices has primarily been confined to theoretical studies, ${ }^{8-12}$ and to date there has only been one experimental demonstration of this mechanism. $^{13}$

Recently, we presented a hybrid nanofabrication process to fabricate multiple, but spatially separated, capless nanotube segments from a single MWNT. ${ }^{14}$ In this paper, we present electrostatically actuated NEMS switches using this construct, which represents an operational demonstration of a bi-directional nanobearing architecture (unidirectional constructs reported previously include $[1-2,13])$. Furthermore, our switches based on ultrasmall bearings ( 200nm length) demonstrate lower actuation voltages (0.8 to $6 \mathrm{~V})$ as compared to those in ref. [13] (where a third of the devices switched below 10V). We also present insights into the repeatability, hysteresis and failure modes of these devices. The hybrid batch fabrication paradigm enables the construction of multiple switches (up to 4 demonstrated here) within a single MWNT at high densites (220nm pitch), and improves on previous efforts that have constructed arc-grown, MWNT-based devices one at a time either by nanorobotic manipulation ${ }^{5,6}$ or by surface micromachining structures at randomly dispersed NT sites on Si chips. ${ }^{3,4}$ 
The key aspects of the nanostructure illustrated in Fig. 1(a) include: (1) Dielectrophoretic (DEP) assembly of a MWNT onto five spatially separated electrodes. The electrodes are designed with a $220 \mathrm{~nm}$ pitch; (2) Piecewise, current-induced shell engineering ${ }^{14,15}$ of the NT to create $\sim 6$ to $15 \mathrm{~nm}$ wide breaks in each of the four inter-electrode NT segments that are suspended in air; (3) The three inner electrodes (labeled 'E2' through 'E4' in Fig. 1(a)) have an open, capless NT segment that is anchored at the outermost shell. As shown in the crosssectional schematic of these NT segments (Fig. 1(b)), the inner shells are free to slide axially within the outer housing in either direction, and (4) at the distal electrodes, the NT caps and the metal electrode constrain the core to axially displace in only one direction, as shown in Fig. 1(c).

The operating principle of the multiple-device configuration contained within a MWNT is illustrated in Fig. 1(d-e). Each pair of spatially separated, opposing nanotube segments located between adjacent metal electrodes constitute a switching device (labeled 'S1' through 'S4' in Fig. 1(a)). In the as-fabricated configuration, all four switches are in the 'OFF' state. When a potential difference is applied between any two adjacent metallic contacts, charges with opposing polarities are induced in the respective nanotube segments. This results in two types of electrostatic forces: (1) an intra-segment repulsive force (due to like charges) that extrudes the core from the outer housing, and (2) an inter-segment attractive force (due to charges with opposing polarities) that causes the core shells within neighboring NT segments to move towards each other. Beyond a characteristic threshold bias, the combined contributions of these two electrostatic force components becomes higher than the van der Waals interactions within each of the two segments, and results in a closure of the gap (Fig. 1(e)). In this 'ON' state, a current flow is established between the metallic contacts.

The batch fabrication process ${ }^{14}$ employed to construct these devices is as follows. Silicon chips with a $500 \mathrm{~nm}$ thick insulating oxide layer on top serve as the substrate. We define a 
lower layer of electrodes $(15 \mathrm{~nm} \mathrm{Cr} / 45 \mathrm{~nm} \mathrm{Au})$ using a combination of electron-beam lithography (EBL) and lift-off. Next, MWNTs are assembled onto the nanoelectrodes from a suspension in ethanol using DEP. We employ a composite-field DEP bias [with a $3.2 \mathrm{~V}$ AC (peak-to-peak) and $0.4 \mathrm{~V}$ DC signal] for 120 seconds. To insure robust electrical and mechanical contact to the NTs, we define a top metallic layer comprising of $15 \mathrm{~nm} \mathrm{Cr} / 45 \mathrm{~nm}$ $\mathrm{Au}$, again using EBL and lift-off. Finally, we employ the current-driven breakdown technique to create nanoscale gaps in each of the inter-electrode, suspended NT segments. In our process, we have observed single NT assembly yields of the order of $40 \%$. This is primarily limited by suspension in-homogeneity, which can be improved with the use of centrifugation in combination with ultrasonication (the use of surfactants is an alternate option but, it negatively impacts on NT conductivity / contact resistance). Detailed information on the fabrication procedure can be found in ref. [14]. Scanning electron microscopy (SEM) images of fabricated NEMS switches are shown in Figure 1(f-g), and a section of the nanoarray design is shown in inset of panel ' $\mathrm{g}$ '. From these images taken with a stage tilt, it is evident that the NT segments are fully suspended in air in the inter-electrode region. In addition, it can be seen that the shell-engineered gaps are located at the mid-lengths of NT segments between electrodes. Altering the location of these etched gaps from NT mid-lengths would enable ultra-precise engineering of bearing lengths. This can potentially be achieved by using nanomachined heat sinks across the NT to control the Joule-heat induced temperature distribution. $^{16}$

During switching experiments involving devices constructed within 13 NTs, the threshold bias for transitions from the 'OFF' to ' $\mathrm{ON}$ ' state was found to vary between 0.8 to $6 \mathrm{~V}$. Current $v s$. voltage plots demonstrating the operation of four supermolecular switches realized within a NT are shown in Fig. 2(a). The voltages required for establishing an 'ON' state in these four devices were found to be in the 0.8 to $2 \mathrm{~V}$ range. This variation in threshold bias can 
be attributed to differences in the inter-segment gaps at each of the four devices. SEM imaging provides direct evidence for inter-shell displacements induced by electrostatic actuation. Fig. 2(b) shows a switch in the 'OFF' state with the gap clearly visible, while panel 'c' shows the gap closed due to electromechanical actuation of the core shells resulting in an 'ON' state.

The repeatability in performance of these devices was experimentally investigated by applying the excitation bias several times to a single device. For the device shown in Fig. 3(a), the 'ON' state current in the first cycle reached a peak of $7.35 \mu \mathrm{A}$ at $2.5 \mathrm{~V}$. Beyond this bias, unexpected step-like decreases in current were observed. These current steps are similar to those previously observed during current driven evaporation of MWNT shells ${ }^{14}$ and indicate a loss of outer NT shells. During the next cycle, the threshold bias for switching increased to $1.7 \mathrm{~V}$ (from $1.1 \mathrm{~V}$ in the first cycle) and the peak current reduced to $4.08 \mu \mathrm{A}$. Apart from current steps in the first cycle, this reduction in 'ON' currents during the second cycle also points to increased device resistance associated with a lower number of shells. However, after the initial reduction in currents, the switching behavior was found to be stable and repeatable as seen in the overlapping plots for cycles 2 and 3. Information on other device and process failure modes can be found in Supplementary Online Information. ${ }^{17}$

Fig. 3(b) represents the hysteresis plot of a device in which the voltage was first increased from 0 to $3 \mathrm{~V}$ and, then, decreased from 3 to $0 \mathrm{~V}$. As can be seen from the measurement, it is evident that the device reaches the ' $\mathrm{ON}$ ' state at $1.8 \mathrm{~V}$ during the increasing voltage segment and returns to the 'OFF' state at $1.2 \mathrm{~V}$ during the decreasing voltage segment. This characteristic can be attributed to electrostatic interactions between the inner shells of the two NT segments, which are a function of both the applied voltage as well as NT position. This component is significantly higher during the decreasing voltage segment (where the inner shells are in proximity at the initial state) as compared to the increasing voltage segment 
(where the inner shells are separated by the fabrication-defined gap of $\sim 6-15 \mathrm{~nm}$ at the initial state).

Furthermore, the increase in current beyond the threshold voltage (without a steep step) is characteristic of tunneling transport at the inter-segment interface, and indicates that the carbon atoms on opposing segments do not re-establish covalent bonds in the 'ON' state. This behavior is different from the previous report on telescoping NEMS switches ${ }^{13}$ in which the IV curve exhibited a step-like increase at the threshold bias and the NT segments remained welded to each other after switching. In switches characterized as a part of our effort, this behavior occurred in only one device (Fig. 3(c)), with the rest exhibiting an I-V profile that indicates tunneling transport at the interface between NT segments during the 'ON' state. This difference arises from the fact that we have characterized our devices primarily in air, and there was no inert ambient created by the flow of Argon, as was the case in ref. [13]. We believe that the difference in inter-segment interface at contact arises from this variation in operating conditions within the two efforts.

We have presented a detailed overview of a low-power, electrostatically actuated NEMS switch based on bi-directional MWNT bearings. The investigation focused on the voltage requirement, repeatability, hysteresis and device failure modes. Further contributions of the work arise from the demonstration of multiple switches within a single MWNT supermolecule, paving the way for high densities.

The authors thank FIRST lab of ETH Zurich for technical support. This work was partially supported by the Swiss Nano-Tera Program and by the Swiss National Science Foundation. 


\section{References}

1. J. Cumings, and A. Zettl, Science 289, 602 (2000).

2. M. F. Yu, B. I. Yakobson, and R. S. Ruoff, J. Phys. Chem. B 104, 8764 (2000).

3. A. M. Fennimore, T. D. Yuzvinsky, W. Q. Han, M. S. Fuhrer, J. Cumings, and A. Zettl, Nature 424, 408 (2003).

4. B. Bourlon, D. C. Glattli, C. Miko, L. Forro, and A. Bachtold, Nano Lett. 4, 709 (2004).

5. L. X. Dong, B. J. Nelson, T. Fukuda, and F. Arai, IEEE Trans. Autom. Sci. Eng. 3, 228 (2006).

6. K. Jensen, C. Girit, W. Mickelson, and A. Zettl, Phys. Rev. Lett. 96, 215503 (2006).

7. S. J. Papadakis, A. R. Hall, P. A. Williams, L. Vicci, M. R. Falvo, R. Superfine, and S. Washburn, Phys. Rev. Lett. 93, 146101 (2004).

8. J. W. Kang, and Q. Jiang, Nanotechnology 18, 095705 (2007).

9. Q. M. Yan, G. Zhou, S. G. Hao, J. Wu, and W. H. Duan, Appl. Phys. Lett. 88, 173107 (2006).

10. L. Maslov, Nanotechnology 17, 2475 (2007).

11. A. M. Popov, E. Bichoutskaia, Y. E. Lozovik, and A. S. Kulish, Phys. Stat. Sol. (A) 204, $1911(2007)$

12. A. S. Kulish, A. M. Popov, Y. E. Lozovik, and E. Bichoutskaia, Full., Nanot. and Carb. Nanost. 16, 340 (2008).

13. V. V. Deshpande, H. Y. Chiu, H. W. C. Postma, C. Miko, L. Forro, and M. Bockrath, Nano Lett. 6, 1092 (2006).

14. A. Subramanian, L. X. Dong, J. Tharian, U. Sennhauser, and B. J. Nelson, Nanotechnology 18, 075703 (2007).

15. I. Yagi, K. Tsukagoshi, E. Watanabe, and Y. Aoyagi, Microelec. Eng. 73-74, 675 (2004). 
16. A. Subramanian, T.-Y. Choi, L. X. Dong, J. Tharian, U. Sennhauser, D. Poulikakos, and B. J. Nelson, App. Phys. A: Mat. Sc. \& Proc. 89, 133 (2007).

17. See EPAPS supplementary material at [URL will be inserted by AIP] for further information on device and process failure modes. 
Figure Captions

FIG. 1. Supermolecular switches based on bi-directional linear bearings. (a) A piecewise engineered MWNT bridging five spatially separated electrodes. The NT is engineered to create gaps in each of the suspended, inter-electrode segments. The labeling terminology for electrodes ('E1' through 'E5') and switches ('S1' through 'S4') that would be used within this report are highlighted here. (b) Bi-directional shell architecture at electrodes 'E2', 'E3', and 'E4'. (c) The shell architecture at electrodes 'E1' and 'E5' showing the unidirectional nature of bearings at these locations. (d) Cross-sectional view of a switch in the 'OFF' state. (e) Cross-sectional view of a switch in the 'ON' state when an excitation bias beyond the characteristic threshold is applied. (f) SEM image (taken with a stage tilt of 40 degrees) of multiple bi-directional bearings constructed within a MWNT using current induced shell etching. The arrows point to gaps engineered at each of the suspended, inter-electrode NT segments. (g) Another MWNT nanostructure with the array design shown in inset. Scale bar represents $100 \mathrm{~nm}$. Image in panel ' $\mathrm{f}$ ' has been reproduced from ref. 14 with permission from IOP Publishing Ltd., Bristol, UK.

FIG. 2. Switching results. (a) I-V plots of switches with low turn-on bias ( 0.8 to $2 \mathrm{~V}$ in this case). The engineered NT is shown in inset. (b-c) SEM images of a device in the open and closed configurations.

FIG. 3. Device repeatability and hysteresis measurements. (a) Evolution of I-V plots during three successive switching cycles. The step-like reduction in currents during the first cycle is attributed to the loss of outer NT shells. The behavior during the second and third cycles remains repeatable, but with a lower 'ON' current as compared to the first cycle. (b) Hysteresis behavior indicated by I-V plots of switches during the increasing and decreasing 
voltage cycles. It can be seen that the 'OFF'-to-'ON' transition $(1.8 \mathrm{~V})$ occurs at a higher voltage as compared to the 'ON'-to-'OFF' transition $(1.2 \mathrm{~V})$. (c) The only device that exhibited an ohmic interface between NT segments in the 'ON' state. 
FIG. 1

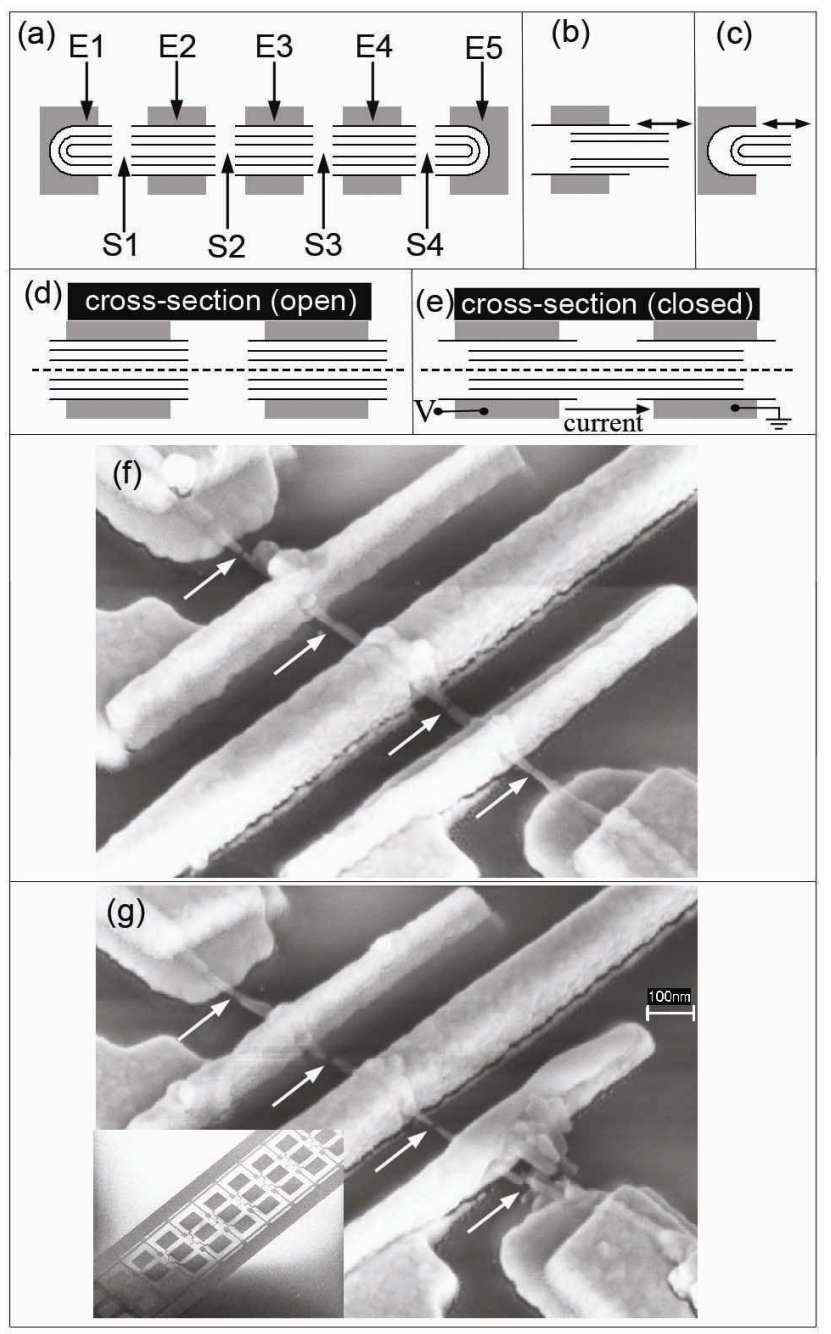

FIG. 2

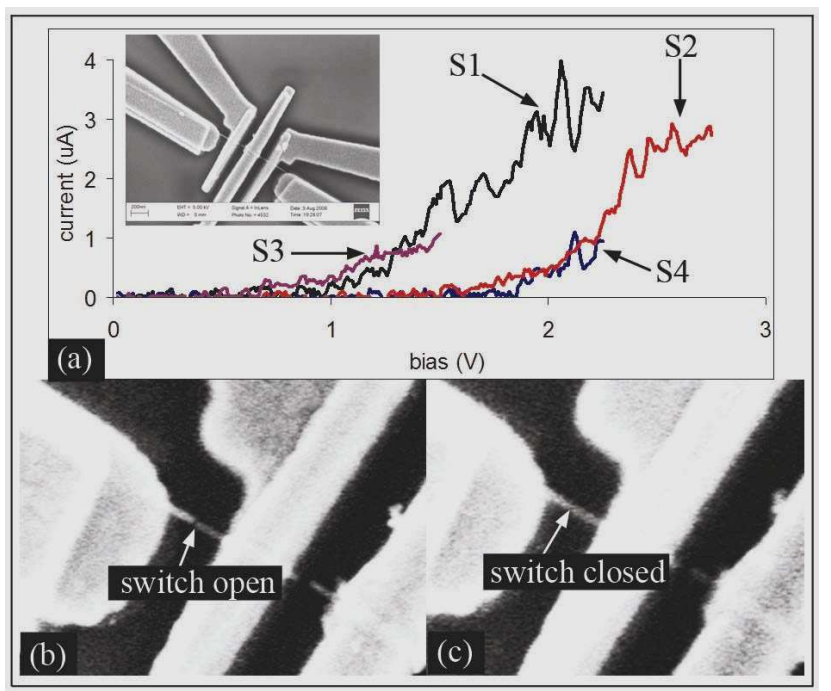


FIG. 3
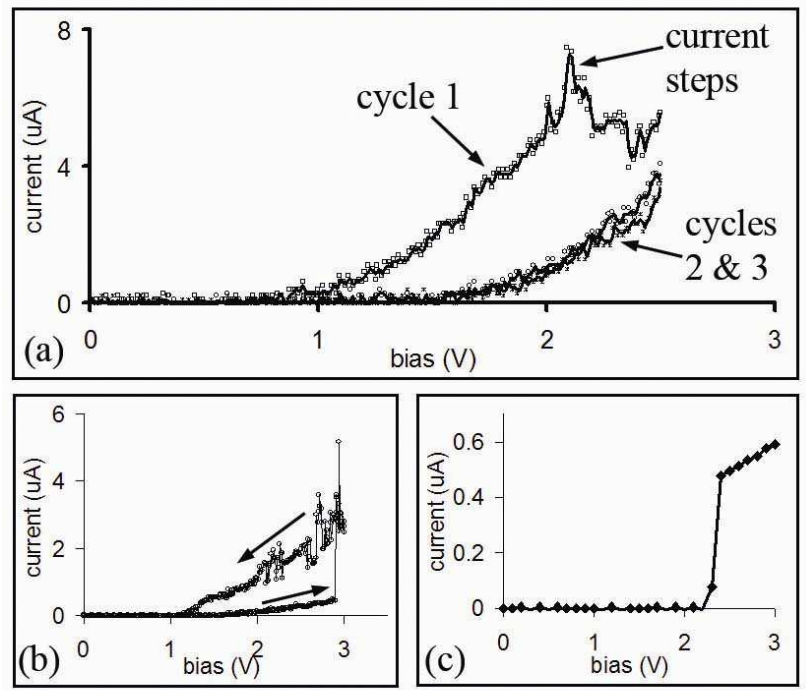


\section{Supplementary Online Information}

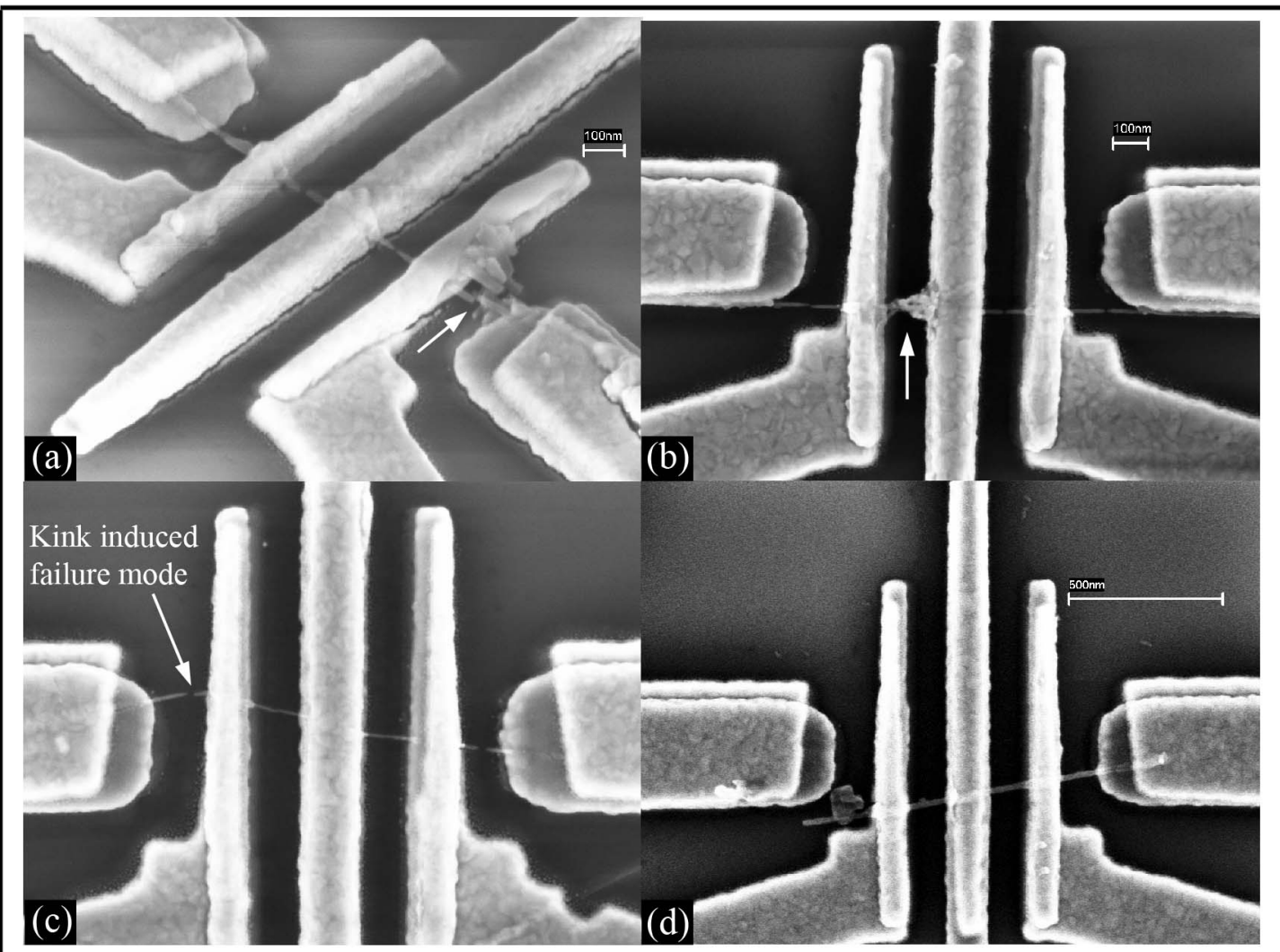

Fig. S1. Device failure modes. (a) Misalignment of opposing NT segments at one of the switches (indicated with an arrow) resulting in device failure. (b) A nanostructure where switching operation at one device location is impeded by the presence of graphitic impurities assembled during DEP. (c) Another NT where a structural imperfection (kink) impedes switching. (d) A device where the NT bridges only four of the five electrodes due to DEP inaccuracy resulting in only three switches (instead of four) after shell engineering.

Out of a total of 13 nanostructures that were tested in our experiments, switching at all four devices within a NT occurred in only one nanostructure (presented in Fig. 2 of manuscript). Of the remaining 12 nanostructures, switching occurred at three devices, two devices and one device (out of a maximum of four possible devices by design) in three, seven and two nanostructures, respectively. This failure to establish switching at some locations is attributed to one of the following reasons: (1) misalignment between the opposing NT segments after shell breakdown, (2) presence of graphitic carbon impurities at device locations that prevent either shell engineering or displacement, (3) presence of structural imperfections such as kinks in the NT, and (4) imperfect assembly during the DEP step resulting in the NT bridging 
only two to four electrodes. Devices representative of these failure modes can be found in Fig. S1. Though the process control capabilities demonstrated within this effort represent the current state-of-the-art, further advances are essential to both prevent these failure modes and to precisely engineer devices such that the tolerance in gap sizes is smaller than one $\mathrm{nm}$. This will result in improved control over device metrics such as threshold bias. 